RESEARCH ARTICLE

\title{
Gauge-invariant 3D Ising actions
}

\author{
R.P.K.C. Malmini Ranasinghe \\ Department of Mathematics, Faculty of Applied Sciences, University of Sri Jayewardenepura, Gangodawila, Nugegoda.
}

Submitted: 11 June 2008; Accepted: 28 July 2008

\begin{abstract}
We formulate gauge-invariant Ising models in three dimension, which describe the open surface version of the Gonihedric Ising models. The energy of these open surfaces does not depend on their area but rather on the number of plaquettes meeting at right angles and the length of boundaries and "seams" where three plaquettes intersect. The models thus describe a gas of open surfaces with rigidity but no bare surface tension. Using mean field techniques, we obtain solutions to zero temperature phase diagram.
\end{abstract}

Keywords: Ising model, mean field theory, open surface solutions.

\section{INTRODUCTION}

In 1971 a gauge invariant self-dual three dimensional Ising, or Z(2) gauge-Higgs model was introduced ${ }^{1}$. It has been suggested that this Hamiltonian might serve as a model of sponge like phases in micro emulsions ${ }^{2}$. In this context the boundaries of the geometrical spin clusters in the model can be thought of as describing a gas of open plaquette surface whose energy depends on both the area and the boundary length. In order to describe open surfaces that have defects like boundaries or seams, it is necessary to introduce Ising variables defined on the links joining nearest neighbouring sites of the dual lattice. When $U_{i j}$ is equal to - 1 , we assume that the dual plaquette to the link $\langle i, j\rangle$ is occupied by the surface, whereas when $U_{i j}$ is equal to +1 there is no surface plaquette dual to the link. The link spins $U_{i j}$ are gauge variables and the model displays a $Z(2)$ gauge invariance.

The general, Hamiltonian can write down in the dual link spins that weights the area, intersections, right angled bends and boundary edges arbitrarily ${ }^{3}$. Recent studies $^{4-7}$, have investigated a class of Ising models containing nearest neighbour, next to nearest neighbour and plaquette terms suggested by Savvidy and Wegner which generate closed surfaces whose weight does not depend on the area at all, but only on the (even) number of plaquettes meeting at right angles ${ }^{8,9}$. These "Gonihedric" Ising models have a free parameter $k$ which gives the relative weight of intersections and right angled bends. For $k>0$ they display a second order phase transition with non-standard critical exponents, whereas at $k=0$ (and probably for $k$ small too) they display a first order transition. The models are interesting in the string theory point of view as a possible alternative discretized random surface action, and from the statistical mechanical point of view because they have zero classical surface tension and a novel flip symmetry for spin planes.

In this paper we investigate an open surface version of the Gonihedric Ising model using mean field techniques. From the string theory perspective this corresponds to introducing "quarks" on the boundaries of the worldsheet and investigating the resulting phase structure. In statistical mechanical terms we are investigating an open surface gas whose classical surface tension is zero, as in the closed surface version, but whose bendings and boundaries carry a non-zero weight.

\section{Definition of the models}

Using the results of an open surface model which assigns zero weight to the area may be written as

$$
\begin{aligned}
H= & \kappa \sum_{<i, j>} U_{i j}-\frac{\kappa}{4} \sum_{<i, j, k, l>}\left(U_{i j} U_{j k}+U_{k j} U_{k l}+U_{k j} U_{j i}+U_{l i} U_{i j}\right) \\
& +\frac{\kappa}{4} \sum_{<i, j, k, l>}\left(U_{i j} U_{j k} U_{k l}+U_{l i} U_{i j} U_{j k}+U_{k l} U_{l i} U_{i j}+U_{j k} U_{k l} U_{l i}\right) \\
& +\frac{(1-\kappa)}{4} \sum_{[i, j, k, l]}\left(U_{i j} U_{k l}+U_{i l} U_{k j}\right)+\left(\lambda-\frac{1+\kappa}{4}\right) \sum_{[i, j, k, l]} U_{i j} U_{j k} U_{k l} U_{l i}
\end{aligned}
$$


where $k$ gives the relative weight of intersections and right angled bends, we have assigned an equal weight $\lambda$ to both boundaries and triple intersections and denoted the plaquette sums explicitly by $[i, j, k, l]$. The energy for a plaquette surface in this model is $E=n_{2}+k n_{4}+\lambda n_{0}$, where $n_{2}$ is the number of links on which plaquettes meet at right angles. $n_{4}$ is the number of links at which four plaquettes meet and $n_{0}$ is the number of links on which an odd number (one or three) plaquettes terminate ${ }^{10}$.

This Hamiltonian may be written in a gaugeinvariant from by introducing Ising spins on the sites and making the substitution $U_{i j} \rightarrow \sigma_{i} U_{i j} \sigma_{j}$ for each link spin. The gauge-invariant transcription of equ.(1) takes the form

$$
\begin{gathered}
H=\kappa \sum_{\langle i, j]} \sigma_{i} U_{i j} \sigma_{j} \\
-\frac{\kappa}{4} \sum_{<i, j, k, l\rangle}\left(\sigma_{i} U_{i j} U_{j k} \sigma_{k}+\sigma_{j} U_{k j} U_{k l} \sigma_{l}+\sigma_{k} U_{k j} U_{j i} \sigma_{i}+\sigma_{l} U_{l i} U_{i j} \sigma_{k}\right) \\
+\frac{\kappa}{4} \sum_{<i, j, k, l\rangle}\left(\sigma_{i} U_{i j} U_{j k} U_{k l} \sigma_{l}+\sigma_{l} U_{l i} U_{i j} U_{j k} \sigma_{k}+\sigma_{k} U_{k l} U_{l i} U_{i j} \sigma_{j}+\sigma_{j} U_{j k} U_{k l} U_{l i} \sigma_{i}\right) \\
+\frac{(1-k)}{4} \sum_{[i, j, k, l]}\left[\left(\sigma_{i} U_{i j} \sigma_{j}\right)\left(\sigma_{k} U_{k l} \sigma_{l}\right)+\left(\sigma_{i} U_{i l} \sigma_{l}\right)\left(\sigma_{j} U_{j k} \sigma_{k}\right)\right]+\left(\lambda-\frac{1+k}{4}\right) \sum_{[i, j, k, l]} U_{i j} U_{j k} U_{k l} U_{l i} .
\end{gathered}
$$

The gauge transformations for the theory are $U_{i j} \rightarrow s_{i} U_{i j} s_{j}$ and $\sigma_{i} \rightarrow s_{i} \sigma_{i}$. The partition function coming from summing over spin configurations $\{U, \sigma\}$ in equ.(2) is identical up to a volume factor $2^{v}$ to that coming from summing over configurations $\{U\}$ in equ.(1). In effect of equ.(1) may be thought of as a unitary gauge-fixed version of equ.(2). In the gauge-invariant formulation a plaquette dual to a link $\langle i, j\rangle$ is present if $\sigma_{i} U_{i j} \sigma_{j}=-1$. The $\sigma_{i}$ spins play the role of matter spins to their gauge counterparts, $U_{i j}$. The planar flip symmetry of the closed surface Gonihedric Ising models is thus promoted to a local gauge symmetry in these open surface gauge-ising models.

The rather fearsome looking Hamiltonian of equ.(2) can be seen to be a close relative of Wegner's original $Z(2)$ gauge-Higgs model

$\left.H=\beta_{1} \sum \underset{\langle i, j\rangle}{\sum\left(\sigma_{i}\right.} U_{i j} \sigma_{j}\right)+\beta_{4} \underset{\langle i, j, k, l\rangle}{\sum} U_{i j} U_{j k} U_{k l} U_{l i}$

when $k=1$ where, schematically, it reduces to the form

$H=\sum_{\langle i, j\rangle} \sigma_{i}\left(U_{i j}+\frac{1}{4} \sum U_{i j}^{3}\right) \sigma_{j}-\frac{1}{4} \sum_{\langle i, j, j\rangle} \sigma_{i}\left(\sum U_{i k}^{2}\right) \sigma_{k}+\left(\lambda-\frac{1}{2}\right) \sum_{[i, j, k, k]} U_{i j} U_{j k} U_{k l} U_{l i} \ldots$

where the outer sums run over nearest neighbours $\langle i, j\rangle$ next to nearest neighbours «i, $k »$ and plaquettes $[i, j, k, l]$ The four $U^{3}{ }_{i j}\left(=U_{i l} U_{l k} U_{k j}\right.$ etc.) terms in the inner nearest neighbour sum connect neighbouring spins by going the long way round the plaquettes containing them and the $U_{i j}^{2}\left(=U_{i j} U_{j k}\right.$ etc.) terms in the inner next to nearest neighbour sum connect the next to nearest neighbour terms in a plaquette. For $k=0$ there is also a considerable simplification

$$
\begin{aligned}
H= & \frac{1}{4} \sum_{[i, j, k, l]}\left[\left(\sigma_{i} U_{i j} \sigma_{j}\right)\left(\sigma_{k} U_{k l} \sigma_{l}\right)+\left(\sigma_{i} U_{i l} \sigma_{l}\right)\left(\sigma_{j} U_{j k} \sigma_{k}\right)\right] \\
& +\left(\lambda-\frac{1}{4}\right) \sum_{[i, j, k, l]}\left(U_{i k} U_{j k} U_{k l} U_{l i}\right)
\end{aligned}
$$

where the first term is a dimmer sum over opposite sides of plaquettes.

We can immediately make some general observations regarding both these actions. Equ.(4) is clearly a "higherderivative" generalization of the standard Z(2) gaugeHiggs model in equ.(3), retaining the lower order terms. Equ.(5) on the other hand replaces the $\sigma U \sigma$ mattergauge couplings by their square. One might argue that the addition of such higher-derivative operators would have no effect on the phase diagram of the gauge-Higgs model, but it is clear that the competing nearest and next to nearest neighbour terms do have an effect in the closed surface case, and it is not unreasonable to expect something similar here.

It is also noteworthy that the purely gauge term can have negative couplings even for positive boundary weights, so antiferromagnetic gauge couplings are physically reasonable. In the limit $\lambda \rightarrow \infty$ boundaries and triple intersections are forbidden and the product of link spins around a plaquette is forced to be one. This constraint is solved by taking $U_{i j}=s_{i} s_{j}$ and takes us back to the original closed surface Gonihedric Hamiltonians for all $k$. Making the gauge transformation $U_{i j} \rightarrow s_{i} U_{i j} s_{j}, \sigma_{i} \rightarrow s_{i} \sigma_{i}$ we see that the partition function in the limit $\lambda \rightarrow \infty$ is simply given by setting all Us equal to one in equ.(2).

Our motivation in this paper is to investigate the action of equs.(4) and (5) in order to study phase structure. Following in the footsteps of we carry out a zerotemperature analysis to identify the ground states using mean field techniques. We defer a more sophisticated analysis using the cluster variational method to a later publication.

\section{Zero Temperature and Mean Field}

Both the zero temperature and mean field analysis of the Hamiltonians in equs.(4) and (5) are based on rewriting the full lattice Hamiltonian as a sum over elementary cube Hamiltonians in order to pick up non-uniform phases, which might be expected to appear here just as they do for the closed surface actions 
$H(\sigma, U)=\sum_{<\mathrm{c}>} h_{c}(\sigma, U)$

Since the whole lattice can be tiled by a given cube configuration we have

$\min H=(\sigma, U)=L^{3} \min h_{c}(\sigma, U)$

and the bulk ground state is obtained by tiling the whole lattice with the cube configuration of U's which minimize the $h_{c}(\sigma, U)$. In some cases there may be several cube configurations minimizing $h_{c}$ and different tiling of the lattice are possible, which gives rise to degenerate ground states.

The single cube Hamiltonian is

$$
\begin{aligned}
& h_{c}\left(\sigma_{i}, U_{i j}\right)=-\frac{k}{4} \sum_{\langle i, j>c}\left(\sigma_{i} U_{i j} \sigma_{j}\right) \\
& +\frac{k}{8} \sum_{\langle i, k>>c C}\left(\sigma_{i} U_{i j} U_{j k} \sigma_{k}+\sigma_{i} U_{i i} U_{l k} \sigma_{k}\right) \\
& -\frac{k}{8} \sum_{\langle i, j>c C}\left(\sigma_{i} U_{i l} U_{l k} U_{k j} \sigma_{j}+\sigma_{i} U_{i m} U_{m n} U_{n j} \sigma_{j}\right) \\
& -\frac{(1-k)}{8} \sum_{[i, j, k, l] \in C}\left[\left(\sigma_{i} U_{i j} \sigma_{j}\right)\left(\sigma_{k} U_{k l} \sigma_{l}\right)+\left(\sigma_{i} U_{i l} \sigma_{l}\right)\left(\sigma_{j} U_{j k} \sigma_{k}\right)\right] \\
& -\left(\frac{\lambda}{2}-\frac{1+k}{8}\right)_{[i, j, k, l] \in C} U_{i j} U_{j k} U_{k l} U_{l i}
\end{aligned}
$$

and one can go to a unitary gauge to analyse the zero temperature energies by fixing all the $\sigma=+1$

$$
\begin{aligned}
& h_{c}\left(\sigma_{i}=1, U_{i j}\right)=-\frac{k}{4} \sum_{<i, j>C C} U_{i j} \\
& +\frac{k}{8} \sum_{<i, k>>C}\left(U_{i j} U_{j k}+U_{i l} U_{l k}\right)-\frac{k}{8} \sum_{<i, j>C C}\left(U_{i l} U_{l k} U_{k j}+U_{i m} U_{m n} U_{n j}\right) \\
& -\frac{(1-k)}{8} \sum_{[i, j, k, l] \in C}\left[U_{i j} U_{k l}+U_{i l} U_{j k}\right]-\left(\frac{\lambda}{2}-\frac{1+k}{8}\right)_{[i, j, k, l, l] C C} U_{i j} U_{j k} U_{k l} U_{l i}
\end{aligned}
$$

For convenience in what follows we denote the coefficient of the pure gauge term as $\tilde{\lambda}$.

$$
\tilde{\lambda}=(\lambda-(1+k) / 4)
$$

If we take $\tilde{\lambda} \rightarrow \infty$ to remove the boundaries and triple intersections we would expect to recover the minimum energy configurations for closed gonihedric surface. As we have noted taking $\tilde{\lambda}, \lambda \rightarrow \infty$ forces the gauge part of the action to be positive and gives $U_{i j}=s_{i} s_{j}$.

For generic $\mathrm{k} \neq 0$ the only state that has the same energy as the (lowest energy) ferromagnetic state with $E=-3(1+k) / 2-3 \tilde{\lambda}$.

Remembering that in the unitary gauge $U=-1$ signifies the presence of a surface plaquette on the dual lattice represents a planar section of surface. Taking $\lambda \rightarrow \infty$ and enforcing $U_{i j}=s_{i} s_{j}$ recovers the layered ground state of the $k \neq 0$ closed surface model.
In mean field approximation the spins are replaced by the average site and link magnetizations. The calculation of the mean field free energy is an elaboration of the zero temperature method in which the energy is still decomposed into a sum of individual cube terms. The mean field theory action is the Hamiltonian plus entropy terms for a single cube, which is minimized by solving a set of 20 coupled equations for the magnetizations in the un-gauge-fixed case or 12 coupled equations in the gauge-fixed case when the site magnetizations are absent. If we denote the average of the variables of $\sigma_{i}$ and $U_{\mathrm{ij}}$ by $m_{i}$ and $l_{i j}$ respectively the mean-field elementary cube free energy is given by

$$
\begin{aligned}
& \Gamma\left(m_{i}, l_{i j}\right)=-\frac{k}{4} \sum_{\langle i, j>c}\left(m_{i} l_{i j} m_{j}\right)
\end{aligned}
$$

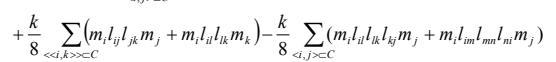

$$
\begin{aligned}
& -\frac{(1-k)}{8} \sum_{[i, j, k, l] \in C}\left[\left(m_{i} l_{i j} m_{j}\right)\left(m_{k} l_{k l} m_{l}\right)+\left(m_{i} l_{i} m_{l}\right)\left(m_{j} l_{j k} m_{k}\right)\right]-\frac{\tilde{\lambda}}{2} \sum_{[i, j, k, l] C C} l_{i j} l_{j k} l_{k l} l_{l i} \\
& +\frac{1}{16 \beta} \sum_{i \subset C}\left[\left(1+m_{i}\right) \ln \left(1+m_{i}\right)+\left(1-m_{i}\right) \ln \left(1-m_{i}\right)\right]+\frac{1}{8 \beta} \sum_{\langle i, j \succ C}\left[\left(1+l_{i j}\right) \ln \left(1+l_{i j}\right)+\left(1-l_{i j}\right) \ln \left(1-l_{i j}\right)\right]
\end{aligned}
$$

The magnetization configuration(s), which minimize the elementary cube free energy is given by

$$
\begin{aligned}
& \frac{\partial \Gamma}{\partial l_{i j(i, j=1 \ldots 12)}}=0 \\
& \frac{\partial \Gamma}{\partial m_{i(i=1 . .8)}}=0
\end{aligned}
$$

These equations simplify somewhat in the special cases $k=0,1$ discussed above, and one can simplify things even further at the expense of losing gauge invariance by going to the unitary gauge and fixing all the $m_{i}$ to one. This then reduces the set of mean field equations to twelve.

More explicitly, when $\mathrm{k}=0$ we have twelve equations of the form

$$
\begin{aligned}
l_{12} & =\tanh \left[\frac{\beta}{2}\left(l_{56}+l_{34}\right)+2 \tilde{\lambda} \beta\left(l_{26} l_{56} l_{15}+l_{23} l_{34} l_{14}\right)\right] \\
& \vdots \\
l_{37} & =\tanh \left[\frac{\beta}{2}\left(l_{26}+l_{48}\right)+2 \tilde{\lambda} \beta\left(l_{34} l_{48} l_{74}+l_{23} l_{26} l_{28}\right)\right]
\end{aligned}
$$

And, similarly, when $\mathrm{k}=1$ we have

$$
\begin{aligned}
l_{12}= & \tanh \left[\beta-\frac{\beta}{2}\left(l_{23}+l_{14}+l_{26}+l_{15}\right)+\frac{\beta}{2}\left(l_{14} l_{23}+l_{15} l_{26}\right)\right. \\
& \left.+2 \bar{\lambda} \beta\left(l_{26} l_{56} l_{15}+l_{23} l_{34} l_{14}\right)\right]
\end{aligned}
$$

equations give solutions for various values of $\tilde{\lambda}$ and $\beta$ to search for phase transitions. In a paramagnetic phase the various $l$ 's will iterate to zero, whereas in a 
pure magnetized phase they will iterate to \pm 1 . Mixed phases are signaled by values of $l$ that are not uniformly 0 or \pm 1 .

\section{DISCUSSION}

Using mean field techniques, we obtain solutions to zero temperature phase diagram for gauge-invariant Ising models in three dimension. This model describes the open surface version of the Gonihedric Ising models introduced by Savvidy and Wegner.

\section{References}

1. Wegner F. (1971). Duality in general Ising models and phase transitions without local order parameters. Journal of Mathematical Physics 12:2259.

2. Huse D. \& Leibler S. (1991). Are sponge phases of membranes experimental gauge-Higgs systems? Physical Review Letters 66:437.

3. Cappi A., Colangelo P., Gonella G. \& Maritan A. (1992). Ensemble of interacting randon surfaces on a lattice.
Nuclear Physics B 370:659.

4. Johnston D.A., Ranasinghe P.K.C. Malmini (1996). Gonihedric 3D ising models. Physics Letters B 378:87.

5. Baig M., Espriu D., Johnston D., Ranasinghe P.K.C. Malmini (1997). Evidence of a first order transition in plaquette 3D Ising like action. Journal of Physics A 30: 405.

6. Baig M., Espriu D., Johnston D.A., Ranasinghe P.K.C. Malmini (1997). String tension in gonihedric threedimensional Ising models. Journal of Physics A 30:7695.

7. Johnston D.A., Lipowski A. \& Ranasinghe P.K.C. Malmini (2008). The gonihedric Ising model and glassiness. Rugged Free-Energy Landscapes - An Introduction (Ed. Janke) Springer Lecture Notes in Physics, Volume 736. pp. 173199.

8. Pietig R. \& Wegner F. (1996). Phase transitions in lattice surface systems with gonihedric action. Nuclear Physics B 466:513.

9. Cirillo E., Gonnella G. \& Pelizzola A. (1997). Critical behavior of the three-dimensional Ising model with nearest-neighbor, next-nearest-neighbor, and plaquette interactions. Physical Review E 55: R17.

10. Savvidy G.K. \& Wegner F. (1994). Geometrical String and spin systems. Journal of Nuclear Physics B 413:605. 